\title{
INEQUALITIES FOR THE ERGODIC MAXIMAL FUNCTION AND CONVERGENCE OF THE AVERAGES IN WEIGHTED $L^{p}$-SPACES ${ }^{1}$
}

\author{
F. J. MARTIN - REYES
}

\begin{abstract}
This paper is concerned with the characterization of those positive functions $w$ such that Hopf's averages associated to an invertible measure preserving transformation $T$ and a positive function $g$ converge almost everywhere for every $f \in L^{p}(w d \mu)$. We also study mean convergence when $g$ satisfies a "doubling condition" over orbits. In order to do this, we first characterize the pairs of positive functions $(u, w)$ such that the ergodic maximal operator associated to $T$ and $g$ is of weak or strong type with respect to the measures $w d \mu$ and $u d \mu$.
\end{abstract}

1. Introduction. Let $(X, \mathscr{F}, \mu)$ be a $\sigma$-finite measure space, let $T$ be an invertible measure preserving transformation from $X$ into itself and let $g$ be a positive function in $L^{1}(d \mu)$.

For each pair of nonnegative integers, $r$ and $k$, we define the operators $D_{r, k}^{g}$, acting on measurable functions, by

$$
D_{r, k}^{g} f(x)=\left[\sum_{i=-r}^{k} f\left(T^{i} x\right)\right]\left[\sum_{i=-r}^{k} g\left(T^{i} x\right)\right]^{-1}
$$

and the maximal operator by

$$
\mathscr{M}_{g} f=\sup _{r, k \geqslant 0} D_{r, k}^{g}|f| \text {. }
$$

Hopf's ergodic theorem applied to $T$ and its inverse transformation $T^{-1}$ assures us that the sequences of averages $\left\{D_{0, k}^{g} f\right\}$ and $\left\{D_{k, 0}^{g} f\right\}$ converge almost everywhere on $X$ for each function $f$ in $L^{1}(d \mu)$. Our aim is to study the a.e. convergence of the sequences $\left\{D_{0, k}^{g} f\right\}$ and $\left\{D_{k, 0}^{g} f\right\}$ with respect to the other measure $w d \mu$, where $w$ is a positive and measurable function. A more explicit statement of the question is the following: "Let us fix $p, 1 \leqslant p<\infty$. Which are the positive and measurable functions $w$ such that for each $f$ in $L^{p}(d \mu)$ the sequences $\left\{D_{0, k}^{g} f\right\}$ and $\left\{D_{k, 0}^{g} f\right\}$

Received by the editors April 23, 1985.

1980 Mathematics Subject Classification. Primary 28D05; Secondary 47A35.

$\mathrm{Ke}$, words and phrases. Ergodic maximal function, mean convergence, almost everywhere convergence, weighted inequalities, Hopf's averages.

'This paper contains some of the results of the author's Doctoral Thesis written under the direction of Professor A. de la Torre at the University of Málaga. 
converge a.e. on $X$ ?" In this paper we characterize those functions by the following conditions:

$$
\begin{array}{cl}
\mathscr{M}_{g}\left(w^{-1 / p-1}\right)(x)<\infty & \text { a.e. for } p>1, \\
\sup _{-\infty<i<\infty} w^{-1}\left(T^{i} x\right)<\infty & \text { a.e. for } p=1 .
\end{array}
$$

To prove these results, we observe, by Theorem 4 in [13], that the a.e. convergence of $\left\{D_{0, k}^{g} f\right\}$ and $\left\{D_{k, 0}^{g} f\right\}$ is equivalent to $\mathscr{M}_{g} f(x)<\infty$ a.e. On the other hand this last problem is related (by Nikishin's theorem [5]) to weak type inequalities for the maximal operator. For that reason we have studied weak and strong type inequalities for $\mathscr{M}_{g}$ in $\$ \S 2-4$. Actually, the results about $\mathscr{M}_{g}$ follow from the study of the maximal operator associated to $T_{r, k}^{g} f=D_{r, k}^{g}(f g)$ which is a bounded operator in $L^{\infty}(d \mu)$ while $\mathscr{M}_{g}$ is not.

2. Weak type inequalities for the maximal operator. Let $(X, \mathscr{F}, \mu)$ and $T$ be as in the previous section. Let $g$ be a measurable positive function, not necessarily in $L^{1}(d \mu)$. For each pair of nonnegative integers, $r$ and $k$, we consider the averages

$$
T_{r, k}^{g} f(x)=D_{r, k}^{g}(f g)(x)=\left[\sum_{i=-r}^{k}(f g)\left(T^{i} x\right)\right]\left[\sum_{i=-r}^{k} g\left(T^{i} x\right)\right]^{-1}
$$

and the maximal operator

$$
M_{g} f=\mathscr{M}_{g}(f g)=\sup _{r, k \geqslant 0} T_{r, k}^{g}|f|,
$$

where $f$ is a measurable function.

In this section we characterize the pairs of nonnegative measurable functions $(u, w)$ satisfying the following property: “ $M_{g}$ is of weak type $(p, p)$ with respect to the measures $w d \mu$ and $u d \mu$." The necessary and sufficient condition on $u$ and $w$ is similar to Muckenhoupt's condition $A_{p}[8]$ for the Hardy-Littlewood maximal operator. For that reason, we will establish $A_{p}$ in our context before stating the theorem (particular cases of our condition can be found in [1 and 2]).

(2.3) Definition. Let $1<p<\infty$ and let $q$ be the conjugate exponent of $p$ (the letter $q$ will always have the same meaning in this paper). The pair $(u, w)$ of nonnegative, measurable real functions satisfies $A_{p}^{\prime}(g, T, \mu)$ (or simply the pair $(u, w)$ belongs to the $A_{p}^{\prime}(g, T, \mu)$ class) if there exists a constant $C>0$ such that for every nonnegative integer $k$ and for a.e. $x[\mu]$

$$
T_{0, k}^{g}\left(g^{-1} u\right)(x)\left[T_{0, k}^{g}\left(w^{-1} g\right)^{q / p}(x)\right]^{p / q} \leqslant C .
$$

Observation. If $T_{0, k}^{g}\left(w^{-1} g\right)^{q / p}(x)=\infty$ (because $w\left(T^{i} x\right)=0$ for some $i, 0 \leqslant i \leqslant k$ ) we assume that (2.4) is satisfied if $T_{0, k}^{g}\left(g^{-1} u\right)(x)=0$, i.e., the pair $(u, w)$ belongs to the $A_{p}^{\prime}(g, T, \mu)$ class if $(2.4)$ is satisfied for a.e. $x$ in $X-\bigcup_{i=-\infty}^{\infty} T^{i}(\{x \in X$ : $w(x)=0\})$ and $\mu(E)=0$, where $E$ is the set

$$
\{x \in X: w(x)=0\}-\bigcap_{i=-\infty}^{\infty} T^{i}(\{x \in X: u(x)=0\}) .
$$


(2.5) Definition. The pair $(u, w)$ of nonnegative, measurable, real functions satisfies $A_{1}^{\prime}(g, T, \mu)$ (or simply the pair $(u, w)$ belongs to the $A_{1}^{\prime}(g, T, \mu)$ class) if there exists a constant $C>0$ such that for a.e. $x[\mu]$

$$
M_{g}\left(g^{-1} u\right)(x) \leqslant C\left(g^{-1} w\right)(x) .
$$

Observations. We shall omit the letters $T$ or $\mu$ in the expression $A_{p}^{\prime}(g, T, \mu)$ when the transformation or the measure we are referring to is clear. We shall not write the function $g$ if $g(x)=1, x \in X$. If the functions $u$ and $w$ are equal we shall simply say that the function $w$ satisfies $A_{p}^{\prime}(g, T, \mu)$.

We shall consider two sets "equal" if they agree up to a set of measure zero.

Throughout this paper, $C$ will denote a positive constant not necessarily the same one at each occurrence.

(2.7) THEOREM. Let $u$ and $w$ be nonnegative measurable real functions. If $1 \leqslant p<$ $\infty$, then the following conditions are equivalent:

(a) There exists $C>0$ such that

$$
\int_{\left\{x \in X: M_{g} f(x)>\lambda\right\}} u d \mu \leqslant C \lambda^{-p} \int_{X}|f|^{p} w d \mu
$$

for every $\lambda>0$ and for each measurable function $f$.

(b) The pair $(u, w)$ satisfies $A_{p}^{\prime}(g, T, \mu)$.

Our main tools to prove this result will be the concept of (ergodic) rectangle and Lemma (2.10).

(2.8) Definition. Let $k$ be a nonnegative integer. The measurable set $B \subset X$ is the base of an (ergodic) rectangle of length $k+1$ if $T^{i} B \cap T^{j} B=\varnothing, i \neq j, 0 \leqslant i$, $j \leqslant k$. In such a case the set $R=\bigcup_{i=0}^{k} T^{i} B$ will be called an (ergodic) rectangle with base $B$ and length $k+1$.

(2.9) LeMma. Let $S$ be a measure preserving transformation from $X$ into itself. For every measurable set $Y \subset X$ there exists a countable family $\left\{B_{i}: i \in \mathbf{Z}^{+}\right\}$of measurable sets such that:

(a) $Y=\bigcup_{i=0}^{\infty} B_{i}$.

(b) $B_{i} \cap B_{j}=\varnothing$ if $i \neq j$.

(c) $S^{-1} B_{i} \cap B_{i}=\varnothing$ if $i \geqslant 1$.

(d) $S^{-1} A=A$ for every subset $A \subset B_{0}$.

(2.10) LEMMA. Let $Y$ be a measurable set of $X$ and let $k$ be a nonnegative integer. Then, there exists a countable family $\left\{B_{i}: i \in \mathbf{Z}^{+}\right\}$of sets of finite measure such that:

(i) $Y=\bigcup_{i=0}^{\infty} B_{i}$.

(ii) $B_{i} \cap B_{j}=\varnothing$ if $i \neq j$.

(iii) For every $i, B_{i}$ is the base of an ergodic rectangle of length $1+s(i)$ with $0 \leqslant s(i) \leqslant k$ and such that if $s(i)<k$, then $T^{1+s(i)} A=A$ for every measurable set $A \subset B_{i}$. Consequently, for every measurable set $A \subset B_{i}$,

$$
\sum_{j=0}^{k} \chi_{T^{i} A} \leqslant C(i) \sum_{j=0}^{s(i)} \chi_{T^{i} A}=C(i) \chi_{\cup_{j=0}^{s(i)} T^{j} A} \leqslant 2 \sum_{j=0}^{k} \chi_{T^{i} A},
$$

where $C(i)$ is the least integer not smaller than $(k+1)(1+s(i))^{-1}$. 
Proof of Lemma (2.9). Let us assume that $\mu(Y)<\infty$. Let $B_{1}=\varnothing$ and let us choose by induction sets $B_{i}$ with $i>1$. Suppose that $B_{1}, B_{2}, \ldots, B_{n}(n \geqslant 1)$ have been chosen. We then define

$$
\mathscr{G}_{n+1}=\left\{B \in \mathscr{F}: B \subset Y, \mu(B)>0, B \cap\left(\bigcup_{i=1}^{n} B_{i}\right)=\varnothing, B \cap S^{-1} B=\varnothing\right\} .
$$

If $\mathscr{G}_{n+1}=\varnothing$ we choose $B_{n+1}=\varnothing$; otherwise we choose a set $B_{n+1} \in \mathscr{G}_{n+1}$ such that $\sup \left\{\mu(B): B \in \mathscr{G}_{n+1}\right\}<2 \mu\left(B_{n+1}\right)$. Finally, let $B_{0}=Y-\bigcup_{i=1}^{\infty} B_{i}$. It is evident that $\left\{B_{i}\right\}_{i=0}^{\infty}$ satisfies (a)-(c). In order to prove that $B_{0}$ satisfies (d) we observe that in the choice of the sets $B_{i}$ we have one of the following situations:

(i) $\mathscr{G}_{m_{0}}=\varnothing$ for some $m_{0} \geqslant 2$,

(ii) $\mathscr{G}_{m} \neq \varnothing$ for every $m \geqslant 2$.

Assume we are in case (i). Let $A$ be any measurable subset of $B_{0}$. It is clear that $\left(A-S^{-1} A\right) \cap\left(\cup_{i=1}^{m_{0}-1} B_{i}\right)=\varnothing$ and $\left(A-S^{-1} A\right) \cap S^{-1}\left(A-S^{-1} A\right)=\varnothing$. Then $\mu\left(A-S^{-1} A\right)=0$ by (i) and since $S$ preserves the measure $\mu$ and $\mu(A)<\infty$ we get that $A=S^{-1} A$.

We now assume that $\mathscr{G}_{m} \neq \varnothing$ for every $m \geqslant 2$. The first thing we notice is that $\lim \mu\left(B_{i}\right)=0$ since $\sum_{i=1}^{\infty} \mu\left(B_{i}\right)=\mu\left(\cup_{i=1}^{\infty} B_{i}\right)=\mu(Y)<\infty$. Let $A$ be any measurable subset of $B_{0}$ and let $D=A-S^{-1} A$. If $\mu(D)>0$, then there exists $n_{0} \geqslant 2$ such that $0<3 \mu\left(B_{n_{0}}\right)<\mu(D)$ and then $3 \mu\left(B_{n_{0}}\right)<\sup \left\{\mu(B): B \in \mathscr{G}_{n_{0}}\right\}$ since $D \in \mathscr{G}_{n_{0}}$. That contradicts the choice of $B_{n_{0}}$. Hence $\mu(D)=0$ and consequently $S^{-1}(A)=A$. This finishes the proof when $\mu(Y)<\infty$.

Now let $Y$ be a measurable subset of $X$. Since $X$ is $\sigma$-finite we have that $Y=\bigcup_{n=0}^{\infty} Y_{n}, Y_{n} \cap Y_{m}=\varnothing$ if $n \neq m$ and $\mu\left(Y_{n}\right)<\infty$. For each $Y_{n}$ we have, by what we have already shown, $Y_{n}=\bigcup_{i=0}^{\infty} B_{i}^{n}$ where $\left\{B_{i}^{n}\right\}_{i}$ is a sequence with the properties (b)-(d) in Lemma (2.9). Then we get the decomposition if $B_{0}=\bigcup_{n=0}^{\infty} B_{0}^{n}$ and the countable family $\left\{B_{i}^{n}: i \geqslant 1, n \in \mathbf{Z}^{+}\right\}$is obtained after renumbering the collection $\left\{B_{j}\right\}_{j=1}^{\infty}$.

Proof of Lemma (2.10). We shall begin by assuming that $\mu(Y)$ is finite. If $k=0$ there is nothing to prove. So, let $k$ be a positive integer. For every $h, 1 \leqslant h \leqslant k$, let $\left\{B_{i, h}\right\}_{i=0}^{\infty}$ be the sequence given by Lemma (2.9) for the set $Y$ and the transformation $T^{-h}$. Then $Y=\bigcap_{h=1}^{k}\left(\cup_{i=0}^{\infty} B_{i, h}\right)=\bigcup_{t \in J} B_{t}$, where $J$ is the set of the mappings from $\{1, \ldots, k\}$ into $\mathbf{Z}^{+}$and $B_{t}=\bigcap_{h=1}^{k} B_{t(h), h}$. It is clear that the collection $\left\{B_{t}\right.$ : $t \in J\}$ is a countable, disjoint family of sets of finite measure. On the other hand, if $\{h: t(h)=0\}=\varnothing$ we have that $B_{t}$ is the base of a rectangle of length $k+1$. If $\{h$ : $t(h)=0\} \neq \varnothing$, it is clear that $B_{t}$ is the base of a rectangle of length $s(t)+1$, where $s(t)+1=\min \{h: t(h)=0\}$ (observe that $s(t)<k$ ). In such a case we get that $T^{s(t)+1} A=A$ for any $A \subset B_{t}$ since $B_{t}$ is a subset of $B_{0, s(t)+1}$. Once these properties have been established we easily deduce (2.11).

Now let $Y$ be any measurable subset of $X$. Since $X$ is $\sigma$-finite, we get that $Y=\bigcup_{n=0}^{\infty} Y_{n}, Y_{n} \cap Y_{m}=\varnothing$ if $n \neq m$ and $\mu\left(Y_{n}\right)<\infty$. For every $Y_{n}$ we consider the decomposition $Y_{n}=\bigcup_{i=0}^{\infty} B_{i}^{n}$ given by Lemma (2.10) in the case of finite measure. It is evident that the family $\left\{B_{i}^{n}: i \in \mathbf{Z}^{+}, n \in \mathbf{Z}^{+}\right\}$satisfies the conditions required in the lemma. 
Before beginning the proof of Theorem (2.7) we shall obtain two corollaries of Lemma (2.10) that generalize Lemma (2.2) in [2].

(2.12) Corollary. Let $(X, \mathscr{F}, \mu)$ be a $\sigma$-finite nonatomic measure space and $T$ an ergodic, invertible measure preserving transformation from $X$ onto itself. Let $Y$ be $a$ subset of $X$. Then for every nonnegative integer $k$ the set $Y$ can be written as a countable union of bases of (ergodic) rectangles of length $k+1$.

Proof of Corollary (2.12). Let $X=\bigcup_{i=0}^{\infty} B_{i}$ be the decomposition given by Lemma (2.10) for $X$ and $k$. To prove the corollary it will suffice to show that $s(i)=k$ for every $B_{i}$ of positive measure. If $s(i)<k$, the set $A \cup T A \cup \cdots \cup T^{s(i)} A$ is invariant for every subset $A$ of $B_{i}$. Hence $\mu\left(A \cup T A \cup \cdots \cup T^{s(i)} A\right)=0$ or $\mu\left(X-A \cup T A \cup \cdots \cup T^{s(i)} A\right)=0$. However both conditions are impossible for a subset $A$ of $B_{i}$ such that $0<\mu(A)<(s(i)+1)^{-1} \mu\left(B_{i}\right)$. Therefore $s(i)=k$ if $\mu\left(B_{i}\right)>0$.

(2.13) Corollary. Let $(X, \mathscr{F}, \mu)$ be a $\sigma$-finite measure space with $\mu(X)=\infty$ and let $T$ be an ergodic, invertible measure preserving transformation from $X$ onto itself. Let $Y$ be a measurable subset of $X$. Then for every nonnegative integer $k$ the set $Y$ can be written as a countable union of bases of (ergodic) rectangles of length $k+1$.

Proof of Corollary (2.13). In order to prove (2.13) we follow the proof of (2.12) taking $A=B_{i}$. Then, since $\mu\left(B_{i}\right)>0$ we have $\mu\left(X-B_{i} \cup \cdots \cup T^{s(i)} B_{i}\right)=0$. But this implies that $\mu(X)<\infty$ because $\mu\left(B_{i}\right)<\infty$. Consequently, $s(i)=k$ if $\mu\left(B_{i}\right)>0$.

Proof of (a) $\Rightarrow$ (b) of Theorem (2.7). Let $1<p<\infty$ and $E=\{x \in X: w(x)=$ $0\}-\bigcap_{i=-\infty}^{\infty} T^{i}(\{x \in X: u(x)=0\})$. Condition (a) with $f=\chi_{E}$ allows us to obtain

$$
\int_{T^{j} E} u d \mu=0 \text { for all integers } j
$$

Thus

$$
\int_{E} \sum_{j=-\infty}^{\infty} u\left(T^{j} x\right) d \mu=0 .
$$

Then $\mu(E)=0$ because $\sum_{j=-\infty}^{\infty} u\left(T^{j} x\right) \neq 0$ if $x \in E$. Now let us fix a nonnegative integer $k$. In order to get (b) we have only to prove that (2.4) is satisfied for a.e. $x$ in the set $F=X-\bigcup_{j=-\infty}^{\infty} T^{j}(\{x \in X: w(x)=0\})$. Let $\left\{B_{i}\right\}_{i=0}^{\infty}$ be the sequence given by Lemma (2.10) for the set $F$ and the number $k$ (in the proof we shall use the notation in Lemma (2.10)). For $B_{i}$ fixed and each integer $m$ we define

$$
H_{i, m}=\left\{x \in B_{i}: 2^{m}<T_{0, k}^{g}\left(g w^{-1}\right)^{q / p}(x) \leqslant 2^{m+1}\right\} .
$$

For any $A \subset H_{i, m}$ set $R=\bigcup_{j=0}^{s(i)} T^{j} A$. Then, if $0 \leqslant h \leqslant k$ and $x \in A$ we have

$$
M_{g}\left(\left(g w^{-1}\right)^{q / p} \chi_{R}\right)\left(T^{h} x\right)>2^{m}
$$


(observe that $R=\bigcup_{j=0}^{k} T^{j} A$ ). Then, (a) allows us to get

$$
\begin{aligned}
\int_{A} \sum_{j=0}^{s(i)} u\left(T^{j} x\right) d \mu & =\int_{R} u d \mu \leqslant C 2^{-m p} \int_{R} g^{q} w^{-q / p} d \mu \\
& =C 2^{-m p} \int_{A} \sum_{j=0}^{s(i)}\left(g^{q} w^{-q / p}\right)\left(T^{j} x\right) d \mu .
\end{aligned}
$$

Therefore, keeping in mind that $A \subset H_{i, m}$, we have

$$
\begin{aligned}
\int_{A} \sum_{j=0}^{k} u\left(T^{j} x\right) d \mu & \leqslant C(i) \int_{A} \sum_{j=0}^{s(i)} u\left(T^{j} x\right) d \mu \\
& \leqslant C 2^{-m p} C(i) \int_{A} \sum_{j=0}^{s(i)}\left(g^{q} w^{-q / p}\right)\left(T^{j} x\right) d \mu \\
& \leqslant 2 C 2^{-m p} \int_{A} \sum_{j=0}^{k}\left(g^{q} w^{-q / p}\right)\left(T^{j} x\right) d \mu \\
& \leqslant 2^{1+p} C \int_{A}\left(\sum_{j=0}^{k}\left(g^{q} w^{-q / p}\right)\left(T^{j} x\right)\right)^{1-p}\left(\sum_{j=0}^{k} g\left(T^{j} x\right)\right)^{p} d \mu
\end{aligned}
$$

Since this inequality is valid for all measurable sets $A \subset H_{i, m}$ and $F=$ $\bigcup_{i=0}^{\infty} \bigcup_{m=-\infty}^{\infty} H_{i, m}$ we conclude that (2.4) is satisfied for a.e. $x$ in $F$ with constant $2^{1+p} C$ independent of $k$. This finishes the proof of the implication (a) $\Rightarrow$ (b) if $p>1$.

Now let $p=1$. Let $k$ be a nonnegative integer and $\left\{B_{i}\right\}_{i=0}^{\infty}$ the family given by Lemma (2.10) for $X$ and $k$. For $B_{i}$ fixed and each integer $m$ we define

$$
H_{i, m}=\left\{x \in B_{i}: 2^{m}<\left[\sum_{j=0}^{k} g\left(T^{j} x\right)\right]^{-1} \leqslant 2^{m+1}\right\} .
$$

For any measurable subset $A$ of $H_{i, m}$ let $R=\bigcup_{j=0}^{s(i)} T^{j} A$. If $x \in A$ and $0 \leqslant h \leqslant k$ we get, by Lemma (2.10)(iii),

$$
M_{g}\left(\chi_{A} g^{-1}\right)\left(T^{h} x\right) \geqslant C(i)\left[\sum_{j=0}^{k} g\left(T^{j} x\right)\right]^{-1} .
$$

Then, since $A \subset H_{i, m}$ we deduce that $R$ is included in $\left\{x \in X: M_{g}\left(\chi_{A} g^{-1}\right)(x)>\right.$ $\left.2^{m} C(i)\right\}$. Therefore, by (a),

$$
\int_{A} \sum_{j=0}^{s(i)} u\left(T^{j} x\right) d \mu \leqslant C 2^{-m}(C(i))^{-1} \int_{A} g^{-1} w d \mu .
$$

Applying (2.11) and keeping in mind that $A \subset H_{i, m}$,

$$
\begin{aligned}
\int_{A} \sum_{j=0}^{k} u\left(T^{j} x\right) d \mu & \leqslant C(i) \int_{A} \sum_{j=0}^{s(i)} u\left(T^{j} x\right) d \mu \leqslant C 2^{-m} \int_{A} g^{-1} w d \mu \\
& \leqslant 2 C \int_{A} g^{-1}(x) w(x) \sum_{j=0}^{k} g\left(T^{j} x\right) d \mu .
\end{aligned}
$$


Now, since $A$ is any measurable subset of $H_{i, m}$ and $X=\bigcup_{i=0}^{\infty} \bigcup_{m=-\infty}^{\infty} H_{i, m}$ we have

$$
T_{0, k}^{g}\left(g^{-1} u\right)(x) \leqslant 2 C g^{-1}(x) w(x) \text { a.e. }
$$

In the same way we get the inequality with $T_{0, k}^{g}$ instead of $T_{0, k}^{g}$. Therefore, $M_{g}\left(g^{-1} u\right)(x) \leqslant 4 C g^{-1}(x) w(x)$ a.e. $x$ in $X$; thus, the pair $(u, w)$ satisfies $A_{1}^{\prime}(g, T, \mu)$.

Proof OF (b) $\Rightarrow$ (a) OF THEOREM (2.7). The proof is a consequence of the result in the integers case. More precisely:

(2.16) Lemma. If the pair $(u, w)$ satisfies $A_{p}^{\prime}(g, T, \mu)$, then there exists $C>0$ such that for a.e. $x$ in $X$, for all $\lambda>0$ and for every mapping $h: \mathbf{Z} \rightarrow \mathbf{R}$,

$$
\sum_{\left\{j \in \mathbf{Z}: m_{g^{x}} h(j)>\lambda\right\}} u\left(T^{j} x\right) \leqslant C \lambda^{-p} \sum_{j=-\infty}^{\infty}|h(j)|^{p} w\left(T^{j} x\right)
$$

where

$$
m_{g^{\prime}} h(j)=\sup _{r, k \geqslant 0}\left[\sum_{i=-r}^{k}|h(j+i)| g\left(T^{j+i} x\right)\right]\left[\sum_{i=-r}^{k} g\left(T^{j+i} x\right)\right]^{-1}, \quad j \in \mathbf{Z} .
$$

Proof OF LemMa (2.16). In order to prove the lemma it will suffice to get inequality (2.17) substituting $m_{g^{x}}$ by the following maximal operators:

$$
\begin{aligned}
& m_{g^{*}}^{*} h(j)=\sup _{k \geqslant 0}\left[\sum_{i=0}^{k}|h(j+i)| g\left(T^{j+i} x\right)\right]\left[\sum_{i=0}^{k} g\left(T^{j+i} x\right)\right]^{-1}, \\
& m_{g^{*}}^{* *} h(j)=\sup _{k \geqslant 0}\left[\sum_{i=0}^{k}|h(j-i)| g\left(T^{j-i} x\right)\right]\left[\sum_{i=0}^{k} g\left(T^{j-i} x\right)\right]^{-1} .
\end{aligned}
$$

The proof of the inequality, being similar for both operators, will be written only for the first.

It follows from $A_{p}^{\prime}(g, T, \mu)$ that, for a.e. $x$ in $X$, if $w\left(T^{i} x\right)=0$ for some integer $i$ then $u\left(T^{j} x\right)=0$ for all integers $j$. Consequently, inequality (2.17) holds for a.e. $x$ in $D=\bigcup_{j=-\infty}^{\infty} T^{j}(\{x \in X: w(x)=0\})$. Now, let $x$ be in $G=X-D$, let $\lambda$ be a positive real number and let $h$ be a mapping from $\mathbf{Z}$ into $\mathbf{R}$. Put $O_{\lambda}=\{j \in \mathbf{Z}$ : $\left.m_{g^{\prime}}^{*} h(j)>\lambda\right\}$ and, for each nonnegative integer $k, O_{\lambda}^{k}=\left\{j \in O_{\lambda}:-k \leqslant j \leqslant k\right\}$. It is clear that there exists a finite family $\left\{I_{r}: r \in \mathscr{J}_{k}\right\}$ of intervals in $\mathbf{Z}$ (consecutive integers sets) such that

(i) $I_{r} \cap I_{s}=\varnothing$ if $r \neq s$,

(ii) $O_{\lambda}^{k} \subset \bigcup_{r \in g_{k}} I_{r}$,

(iii) $\sum_{j \in I_{r}}|h(j)| g\left(T^{i} x\right)>\lambda \sum_{j \in I_{r}} g\left(T^{j} x\right)$.

Then,

$$
\begin{aligned}
\sum_{j \in O_{\lambda}^{k}} u\left(T^{j} x\right) & \leqslant \sum_{r \in \mathscr{I}_{k}} \sum_{j \in I_{r}} u\left(T^{j} x\right) \\
& \leqslant \lambda^{-p} \sum_{r \in \mathscr{J}_{k}}\left[\sum_{j \in I_{r}}|h(j)| g\left(T^{j} x\right)\right]^{p}\left[\sum_{j \in I_{r}} g\left(T^{j} x\right)\right]^{-p} \sum_{j \in I_{r}} u\left(T^{j} x\right) .
\end{aligned}
$$


If $p=1$ it follows from $(2.21)$ and $A_{1}^{\prime}(g, T, \mu)$ that

$$
\sum_{j \in O_{\lambda}^{k}} u\left(T^{j} x\right) \leqslant \lambda^{-1} C \sum_{r \in \mathscr{J}_{k}} \sum_{j \in I_{r}}|h(j)| w\left(T^{j} x\right) \text { a.e. } x \text { in } G .
$$

Letting $k$ go to $\infty$ we obtain (2.17) for a.e. $x$ in $G$, with $p=1$ and $m_{g^{*}}^{*}$ instead of $m_{g}$.

If $p>1$ we apply Hölder's inequality in the last member of (2.21). Then, $A_{p}^{\prime}(g, T, \mu)$ gives us

$$
\sum_{j \in O_{\lambda}^{k}} u\left(T^{j} x\right) \leqslant C \lambda^{-p} \sum_{r \in \mathscr{Z}_{k}} \sum_{j \in I_{r}}|h(j)|^{p} w\left(T^{j} x\right)
$$

for a.e. $x$ in $G$. Letting $k$ go to $\infty$ we obtain (2.17) for a.e. $x$ in $G$, with $p>1$ and $m_{g^{\prime}}^{*}$ instead of $m_{g^{\prime}}$.

Once Lemma (2.16) has been shown we shall prove implication (b) $\Rightarrow$ (a) of Theorem (2.7) using transference methods.

Let $f$ be a measurable function from $X$ into $\mathbf{R}$ and consider the truncated maximal operator

$$
M_{g, L} f=\sup _{r+k+1 \leqslant I .} T_{r, k}^{g}|f|,
$$

where $L$ is an integer bigger than or equal to 1 . Let us fix $\lambda>0$ and denote by $O_{\lambda, L}$ the set $\left\{x \in X: M_{g, L} f(x)>\lambda\right\}$. Then, since $T$ preserves the measure $\mu$ we have

$$
\int_{O_{\lambda, I .}} u d \mu=(2 k+1)^{-1} \sum_{j=-k}^{k} \int_{T^{-1} O_{\lambda, L .}} u\left(T^{j} x\right) d \mu .
$$

If $[-k-L, k+L]$ denotes the interval in the integers $\{i \in \mathbf{Z}:-k-L \leqslant i \leqslant k+L\}$ and $f^{x}(j)=f\left(T^{j} x\right)$, then the right-hand side of (2.22) is not bigger than

$$
\int_{X}(2 k+1)^{-1} \sum u\left(T^{j} x\right) d \mu
$$

where the sum is extended over the integers $j$ such that $m_{g^{x}}\left(f^{x} \chi_{[-k-L, k+L]}\right)(j)>\lambda$. By Lemma (2.16), the integrand is smaller than or equal to

$$
C(2 k+1)^{-1} \lambda^{-p} \sum_{j=-k-L}^{k+L}\left|f^{x}(j)\right|^{p} w\left(T^{j} x\right) .
$$

If we put these inequalities in (2.22) we have

$$
\int_{O_{\lambda, I}} u d \mu \leqslant C \lambda^{-p}(2 k+2 L+1)(2 k+1)^{-1} \int_{X}|f|^{p} w d \mu .
$$

Now, letting $k$ and then $L$ go to $\infty$ we obtain condition (a) in Theorem (2.7).

(2.24) CoROllary. The maximal operator $M_{g}$ is of weak type $(1,1)$ and strong type $(p, p)$ with respect to the measure $g d \mu$ for every $p, 1<p \leqslant \infty$, with constants independent of $g$.

The corollary follows from Theorem (2.7) by interpolation, since $g$ satisfies $A_{1}^{\prime}(g, T, \mu)$ with constant $C=1$ and $M_{g}$ is of type $(\infty, \infty)$ (also with constant $C=1)$ with respect to $g d \mu$. 
As we shall see later, the weak type $(p, p)$ of the maximal operator is not equivalent to the strong type $(p, p)$. However, if $g$ satisfies a "doubling condition," i.e., if there exists $C_{g}>0$ such that for all nonnegative integers $k$ and for almost everywhere $x$ in $X$,

$$
\sum_{i=-k}^{2 k} g\left(T^{i} x\right) \leqslant C_{g} \sum_{i=0}^{k} g\left(T^{i} x\right)
$$

then the conditions in Theorem (2.7) are equivalent to the uniform boundedness and to the uniform weak type of the averages.

(2.26) THEOREM. If $g$ satisfies the "doubling condition" (2.25), then conditions (a) and (b) in Theorem (2.7) are equivalent to the following conditions (c) and (d).

(c) There exists $C>0$ such that for every $\lambda>0$ and for all measurable functions $f$

$$
\sup _{k \geqslant 0} \int_{\left\{x \in X:\left|T_{k, k}^{g} f(x)\right|>\lambda\right\}} u d \mu \leqslant C \lambda^{-p} \int_{X}|f|^{p} w d \mu .
$$

(d) There exists $C>0$ such that for all measurable functions $f$

$$
\sup _{k \geqslant 0} \int_{X}\left|T_{k, k}^{g} f\right|^{p} u d \mu \leqslant C \int_{X}|f|^{p} w d \mu .
$$

Proof of TheOrem (2.26). It will suffice to prove (c) $\Rightarrow(b)$ and $(b) \Rightarrow(d)$ because (d) $\Rightarrow$ (c) is obvious.

Assume (c) with $p>1$. As in Theorem (2.7) it is clear that $\mu(E)=0$ where

$$
E=\{x \in X: w(x)=0\}-\bigcap_{i=-\infty}^{\infty} T^{i}(\{x \in X: u(x)=0\}) .
$$

Let us fix $k$ and let $B_{i}, H_{i, m}, A$ and $R$ be as in the proof of (a) $\Rightarrow$ (b) in Theorem (2.7) for $p>1$. Then, if $x \in A$ and $0 \leqslant h \leqslant k$

$$
T_{k, k}^{g}\left(\left(w^{-1} g\right)^{q / p} \chi_{R}\right)\left(T^{h} x\right) \geqslant \sum_{i=0}^{k}\left(g^{q} w^{-q / p}\right)\left(T^{i} x\right)\left[\sum_{i=-k}^{2 k} g\left(T^{i} x\right)\right]^{-1} .
$$

Since $g$ satisfies (2.25), the right-hand side of the inequality is bigger than or equal to

$$
\left(C_{g}\right)^{-1} T_{0, k}^{g}\left(w^{-1} g\right)^{q / p}(x) .
$$

Now, keeping in mind that $x \in A \subset H_{i, m}$, we obtain

$$
T_{k, k}^{g}\left(\left(w^{-1} g\right)^{q / p} \chi_{R}\right)\left(T^{h} x\right)>\left(C_{g}\right)^{-1} 2^{m}, \quad 0 \leqslant h \leqslant k, x \in A .
$$

Then, by (c),

$$
\begin{aligned}
\int_{A} \sum_{j=0}^{s(i)} u\left(T^{j} x\right) d \mu & =\int_{R} u d \mu \\
& \leqslant C\left(C_{g}\right)^{p} 2^{-m p} \int_{A} \sum_{j=0}^{s(i)}\left(g^{q} w^{-q / p}\right)\left(T^{j} x\right) d \mu
\end{aligned}
$$


This last inequality is similar to (2.14) with the constant $C\left(C_{g}\right)^{p}$ instead of $C$. Then condition (b) $(p>1)$ follows from (2.27) in the same way that (b) $(p>1)$ is obtained from (2.14) in Theorem (2.7).

Now, assume (c) with $p=1$. Let us fix $k$ and let $B_{i}, H_{i, m}, A$ and $R$ be as in the proof of (a) $\Rightarrow(\mathrm{b})$ in Theorem (2.7) for $p=1$. Then if $x \in A$ and $0 \leqslant h \leqslant k$ we have

$$
T_{k, k}^{g}\left(\chi_{A} g^{-1}\right)\left(T^{h} x\right) \geqslant C(i)\left[\sum_{j=-k}^{2 k} g\left(T^{j} x\right)\right]^{-1} .
$$

Since $g$ satisfies (2.25), the right-hand side of the inequality is bigger than or equal to $C(i)\left(C_{g} \sum_{j=0}^{k} g\left(T^{j} x\right)\right)^{-1}$. Keeping in mind that $A$ is included in $H_{i, m}$ we obtain that $R$ is a subset of $\left\{x \in X: T_{k, k}^{g}\left(\chi_{A} g^{-1}\right)(x)>2^{m} C(i)\left(C_{g}\right)^{-1}\right\}$. Then (c) $(p=1)$ gives us

$$
\int_{A} \sum_{j=0}^{s(i)} u\left(T^{j} x\right) d \mu \leqslant C C_{g}(C(i))^{-1} 2^{-m} \int_{A} g^{-1} w d \mu .
$$

This last inequality is similar to (2.15) with constant $C C_{g}$ instead of $C$. Then, condition (b) $(p=1)$ follows from (2.28) in the same way that (b) $(p=1)$ is obtained from (2.15) in Theorem (2.7).

We shall now prove that $A_{p}^{\prime}(g, T, \mu)$ implies (d). We shall begin with the case $p=1$. Let $f: X \rightarrow \mathbf{R}$ be a measurable function and let $k$ be a nonnegative integer. It is clear that

$$
\int_{X}\left|T_{k, k}^{g} f\right| u d \mu \leqslant \sum_{i=-k}^{k} \int_{X}(|f| g)\left(T^{i} x\right)\left[\sum_{j=-k}^{k} g\left(T^{j} x\right)\right]^{-1} u(x) d \mu
$$

and since $T$ preserves the measure $\mu$

$$
\int_{X}\left|T_{k, k}^{g} f\right| u d \mu \leqslant \int_{X}(|f| g)(x) \sum_{i=-k}^{k}\left[\sum_{j=-k}^{k} g\left(T^{j+i} x\right)\right]^{-1} u\left(T^{i} x\right) d \mu .
$$

The right-hand side in the inequality is smaller than or equal to

$$
C(g) \int_{X}(|f| g)(x) T_{k, k}^{g}\left(g^{-1} u\right)(x) d \mu
$$

since $g$ satisfies (2.25), where $C(g)$ is a constant depending only on $g$. Thus, with this observation and (2.29) we have

$$
\int_{X}\left|T_{k, k}^{g} f\right| u d \mu \leqslant C(g) \int_{X}|f| g M_{g}\left(g^{-1} u\right) d \mu .
$$

Then condition (d) (in the case $p=1$ ) follows from (2.31) since the pair $(u, w)$ satisfies $A_{1}^{\prime}(g, T, \mu)$.

Now, let $p>1$ and assume that the pair $(u, w)$ satisfies $A_{p}^{\prime}(g, T, \mu)$. Let $f$ be a measurable function and let $k$ be a nonnegative integer. Then $A_{p}^{\prime}(g, T, \mu)$ gives us

$$
\int_{X}\left|T_{k, k}^{g} f\right|^{p} u d \mu=\int_{F}\left|T_{k, k}^{g} f\right|^{p} u d \mu
$$


where $F=X-\bigcup_{i=-\infty}^{\infty} T^{i}(\{x \in X: w(x)=0\})$. By Hölder's inequality it is clear that

$$
\left|T_{k, k}^{g} f\right|^{p}(x) \leqslant T_{k, k}^{g}\left(|f|^{p} g^{-1} w\right)(x)\left[T_{k, k}^{g}\left(w^{-1} g\right)^{q / p}(x)\right]^{p / q} \quad(x \in F) .
$$

Hence, keeping in mind that $T$ preserves the measure,

$$
\begin{aligned}
\int_{X}\left|T_{k, k}^{g} f\right|^{p} u d \mu \leqslant \int_{F}|f|^{p}(x) w(x)\left[\sum_{i=-k}^{k}\left[T_{k, k}^{g}\left(w^{-1} g\right)^{q / p}\left(T^{i} x\right)\right]^{p / q}\right. \\
\left.\cdot\left[\sum_{j=-k}^{k} g\left(T^{j+i} x\right)\right]^{-1} u\left(T^{i} x\right)\right] d \mu .
\end{aligned}
$$

Now, from (2.25) it follows that

$$
\begin{aligned}
\sum_{i=-k}^{k}\left[T_{k, k}^{g}\left(w^{-1} g\right)^{q / p}\left(T^{i} x\right)\right]^{p / q}\left[\sum_{j=-k}^{k} g\left(T^{j+i} x\right)\right]^{-1} u\left(T^{i} x\right) \\
\leqslant(C(g))^{p}\left[T_{2 k, 2 k}^{g}\left(w^{-1} g\right)^{q / p}(x)\right]^{p / q} T_{2 k, 2 k}^{g}\left(g^{-1} u\right)(x)
\end{aligned}
$$

and since the pair $(u, w)$ satisfies $A_{p}^{\prime}(g, T, \mu)$, inequalities (2.32) and (2.33) give us

$$
\int_{X}\left|T_{k, k}^{g} f\right|^{p} u d \mu \leqslant C(C(g))^{p} \int_{X}|f|^{p} w d \mu
$$

which proves $(\mathrm{d})$.

(2.34) Note. It is easy to see that, under the assumptions of Theorem (2.26), conditions $(c)$ and $(d)$ are equivalent to $\left(c^{\prime}\right)$ and $\left(d^{\prime}\right)$ :

(c') There exists $C>0$ such that for all $\lambda>0$ and for every measurable function $f$

$$
\sup _{r, k \geqslant 0} \int_{\left\{x \in X:\left|T_{r, k}^{g} f(x)\right|>\lambda\right\}} u d \mu \leqslant C \lambda^{-p} \int_{X}|f|^{p} w d \mu \text {. }
$$

(d') There exists $C>0$ such that for every measurable function $f$

$$
\sup _{r, k \geqslant 0} \int_{X}\left|T_{r, k}^{g} f\right|^{p} u d \mu \leqslant C \int_{X}|f|^{p} w d \mu .
$$

(2.35) EXAmpLe. If $g$ does not satisfy (2.25), then Theorem (2.26) does not hold. In order to observe this, we consider $X=\mathbf{Z}$, the set of the integers, $\mathscr{F}$ the $\sigma$-algebra whose elements are all the subsets of $\mathbf{Z}, \mu$ the counting measure, $T(i)=i+1$, $w(i)=1, u=w$ and $g(i)=2^{i}$. It is not difficult to prove that the averages are uniformly bounded in $L^{p}(w d \mu)$ for every $p, 1 \leqslant p<\infty$. Nevertheless $w$ does not satisfy $A_{p}^{\prime}(g, T, \mu)$ for any $p$. In consequence $M_{g}$ is not of weak type $(p, p)$ for any p.

3. Strong type inequalities for the maximal operator. In this section we want to study, under the same hypothesis as in the previous section: Which condition or conditions must the functions $u$ and $w$ satisfy for the maximal operator $M_{g}$ to be of strong type $(p, p)(1<p<\infty)$, i.e., when is it true that there exists a constant $C>0$ such that

$$
\int_{X}\left|M_{g} f\right|^{p} u d \mu \leqslant C \int_{X}|f|^{p} w d \mu
$$


for every measurable function $f$ ? First of all, it is clear by Theorem (2.7) that a necessary condition (for $M_{g}$ to be of strong type $(p, p)$ ) is that the pair $(u, w)$ satisfies $A_{p}^{\prime}(g, T, \mu)$. Then it is natural to ask if the $A_{p}^{\prime}(g, T, \mu)$ condition is sufficient. The answer is affirmative when $u=w$ (Theorem (4.1)) but in the general case is negative as the following example shows.

(3.1) ExAmple. Let $X, \mathscr{F}, \mu$ and $T$ be as in Example (2.35). We consider $g(i)=1, u(i)=1+|i|$ and $w(i)=(1+|i|)^{2}$. An easy computation proves that the pair $(u, w)$ satisfies $A_{2}^{\prime}(g, T, \mu)$. Nevertheless, if $f=\chi_{\{0\}}$ (characteristic function of the set $\{0\})$ we have that $M_{g} f=u^{-1}$ and

$$
\sum_{i=-\infty}^{\infty}\left|M_{g} f(i)\right|^{2} u(i)=\sum_{i=-\infty}^{\infty} u^{-1}(i)=\infty
$$

while $\sum_{i=-\infty}^{\infty}|f(i)|^{2} w(i)=1$ and hence $M_{g}$ is not of strong type (2,2).

In order to find some necessary and sufficient condition on the functions $u$ and $w$ for the operator to be of strong type $(p, p)(1<p<\infty)$ with respect to the measures $w d \mu$ and $u d \mu$ we look at Sawyer's condition [11 and 12] which characterizes the strong type $(p, p)$ of the Hardy-Littlewood maximal operator. Before stating and proving the main result of this section we must establish which is condition $S_{p}$ in our context.

(3.2) Definition. Let $r$ and $k$ be nonnegative integers. For each measurable function $f: X \rightarrow \mathbf{R}$ we define $M_{r, k}^{g} f$ by

$$
M_{r, k}^{g} f=\max _{\substack{0 \leqslant h \leqslant r \\ 0 \leqslant j \leqslant k}} T_{h, j}^{g}|f| .
$$

(3.3) Definition. Let $1<p<\infty$. The pair $(u, w)$ of nonnegative measurable real functions satisfies $S_{p}^{\prime}(g, T, \mu)$ (or simply the pair $(u, w)$ is in the $S_{p}^{\prime}(g, T, \mu)$ class) if

$$
\mu\left(\{x \in X: w(x)=0\}-\bigcap_{i=-\infty}^{\infty} T^{i}(\{x \in X: u(x)=0\})\right)=0
$$

and there exists $C>0$ such that for all nonnegative integers $k$ and for a.e. $x$ in $X-\bigcup_{i=-\infty}^{\infty} T^{i}(\{x \in X: w(x)=0\})$

$$
\sum_{i=0}^{k}\left|M_{i, k-i}^{g}\left(w^{-1} g\right)^{q / p}\left(T^{i} x\right)\right|^{p} u\left(T^{i} x\right) \leqslant C \sum_{i=0}^{k}\left(g^{q} w^{-q / p}\right)\left(T^{i} x\right) .
$$

With these notations and definitions we have

(3.5) THEOREM. If $1<p<\infty$ the following are equivalent:

(a) There exists $C>0$ such that for every measurable function,

$$
\int_{X}\left|M_{g} f\right|^{p} u d \mu \leqslant C \int_{X}|f|^{p} w d \mu .
$$

(b) The pair $(u, w)$ satisfies $S_{p}^{\prime}(g, T, \mu)$.

Proof of Theorem (3.5). Suppose that proposition (a) is satisfied. As in Theorem (2.7) we prove that $\mu(E)=0$, where $E$ is the set

$$
\{x \in X: w(x)=0\}-\bigcap_{i=-\infty}^{\infty} T^{i}(\{x \in X: u(x)=0\}) .
$$


Then, in order to prove (b) we have only to show that (3.4) is satisfied for almost all $x$ in $F=X-\cup_{i=-\infty}^{\infty} T^{i}(\{x \in X: w(x)=0\})$. Let us fix a nonnegative integer $k$ and let $\left\{B_{i}\right\}_{i=0}^{\infty}$ be the sequence in Lemma (2.10) for the set $F$ and for the number $k$ (in the proof we shall use the notation introduced in Lemma (2.10)). Let us fix a set $B_{i}$. Let $A$ be a measurable subset of $B_{i}$ and define $R=\bigcup_{j=0}^{s(i)} T^{j} A$. Then, if $0 \leqslant h \leqslant k$ and $x \in A$ we have

$$
M_{h, k-h}^{g}\left(w^{-1} g\right)^{q / p}\left(T^{h} x\right) \leqslant M_{g}\left(\left(w^{-1} g\right)^{q / p} \chi_{R}\right)\left(T^{h} x\right) .
$$

Then, (3.6) and the properties of the sets $B_{i}$ give us

$$
\begin{aligned}
\int_{A} \sum_{h=0}^{k} \mid M_{h, k-h}^{g} & \left.\left(w^{-1} g\right)^{q / p}\left(T^{h} x\right)\right|^{p} u\left(T^{h} x\right) d \mu \\
& \leqslant C(i) \int_{R}\left|M_{g}\left(w^{-1} g\right)^{q / p} \chi_{R}\right|^{p} u d \mu .
\end{aligned}
$$

Now, (a) and (3.7) allow us to get

$$
\begin{aligned}
& \int_{A} \sum_{h=0}^{k}\left|M_{h, k-h}^{g}\left(w^{-1} g\right)^{q / p}\left(T^{h} x\right)\right|^{p} u\left(T^{h} x\right) d \mu \\
& \quad \leqslant C C(i) \int_{R} g^{q} w^{-q / p} d \mu \leqslant 2 C \int_{A} \sum_{h=0}^{k}\left(g^{q} w^{-q / p}\right)\left(T^{h} x\right) d \mu,
\end{aligned}
$$

where the last inequality is a consequence of the properties of the sets $B_{i}$. Since $A$ is any measurable subset of $B_{i}$ it follows from (3.8) that

$$
\sum_{h=0}^{k}\left|M_{h, k-h}^{g}\left(w^{-1} g\right)^{q / p}\left(T^{h} x\right)\right|^{p} u\left(T^{h} x\right) \leqslant 2 C \sum_{h=0}^{k}\left(g^{q} w^{-q / p}\right)\left(T^{h} x\right)
$$

for almost all $x$ in $B_{i}$. This finishes the proof of $(a) \Rightarrow(b)$ if we keep in mind that $F=\bigcup_{i=0}^{\infty} B_{i}$ and $k$ is any nonnegative integer.

The other implication $(b) \Rightarrow$ (a) follows from the result in the case of the integers. More precisely:

(3.9) Lemma. Let $1<p<\infty$. If the pair $(u, w)$ satisfies $S_{p}^{\prime}(g, T, \mu)$, then there exists $C>0$ such that for almost all $x$ in $X$ and for every function $h: \mathbf{Z} \rightarrow \mathbf{R}$

$$
\sum_{j=-\infty}^{\infty}\left|m_{g^{\prime}} h(j)\right|^{p} u\left(T^{j} x\right) \leqslant C \sum_{j=-\infty}^{\infty}|h(j)|^{p} w\left(T^{j} x\right) .
$$

The proof of this lemma follows the lines of Sawyer's theorem [11, 12] for the Hardy-Littlewood maximal function. For that reason, we shall only point up some steps of the proof.

As in Lemma (2.16), it suffices to get inequality (3.10) for the operator $m_{g^{x}}^{*}$ instead of $m_{g^{x}}$. First, observe that inequality (3.10) is obvious for almost all $x$ in $\bigcup_{j=-\infty}^{\infty} T^{j} E$, where $E=\{x \in X: w(x)=0\}$. Consider now a nonnegative integer $L$ and the truncated maximal operator

$$
m_{g^{\prime}, L}^{*} h(j)=\sup _{0 \leqslant k \leqslant L} \sum_{i=0}^{k}|h(j+i)| g\left(T^{j+i} x\right)\left[\sum_{i=0}^{k} g\left(T^{j+i} x\right)\right]^{-1} .
$$


Let $x$ be in $F=X-\cup_{j=-\infty}^{\infty} T^{j} E$ and let us fix a function $h: \mathbf{Z} \rightarrow \mathbf{R}$. Let us define for every integer $k$ the set $O_{k}=\left\{j \in \mathbf{Z}: m_{g^{x}, L}^{*}\left(h \chi_{[-L, L]}\right)(j)>2^{k}\right\}$, where $[-L, L]$ $=\{j \in \mathbf{Z}:-L \leqslant j \leqslant L\}$. It is clear that $O_{k}$ is a finite set. Finally, denote by $\left\{I_{r, k}\right.$ : $\left.r \in \mathscr{J}_{k}\right\}$ the family of maximal intervals contained in $O_{k}$. These intervals have the following properties:

(i) $O_{k}=\bigcup_{r \in \mathscr{J}_{k}} I_{r, k} \subset[-2 L, 2 L]$.

(ii) $I_{r, k} \cap I_{s, k}=\varnothing$ if $r \neq s$.

(iii) $2^{k} \sum_{i \in I_{r, k}} g\left(T^{i} x\right)<\sum_{i \in I_{r, k}}|h(i)| g\left(T^{i} x\right)$.

(iv) If $j<k$, then for every interval $I_{r, k}$ there exists $I_{s, j}$ such that $I_{r, k} \subset I_{s, j}$.

From now on, the intervals $I_{r, k}$ play the role of the dyadic cubes in Sawyer's theorem. Then, writing Sawyer's proof with these intervals we get that there exists a constant $C>0$ such that for almost all $x$ in $F$

$$
\sum_{j=-\infty}^{\infty}\left|m_{g^{x}, L}^{*}\left(h \chi_{[-L, L]}\right)(j)\right|^{p} u\left(T^{j} x\right) \leqslant C \sum_{j=-\infty}^{\infty}|h(j)|^{p} w\left(T^{j} x\right) .
$$

If we let $L$ go to $\infty$ we have (3.10) with $m_{g^{x}}^{*}$ instead of $m_{g^{x}}$ for almost all $x$ in $F$, and so the proof of the lemma is finished.

Once Lemma (3.9) is established we shall prove implication (b) $\Rightarrow$ (a) of Theorem (3.5). Let $f: X \rightarrow \mathbf{R}$ be a measurable function and consider the truncated maximal operator $M_{g, L}(L \geqslant 1)$

$$
M_{g, L} f=\sup _{r+k+1 \leqslant L .} T_{r, k}^{g}|f| .
$$

Since $T$ preserves the measure $\mu$, we have

$$
\int_{X}\left|M_{g, L} f\right|^{p} u d \mu=(2 k+1)^{-1} \sum_{j=-k}^{k} \int_{X}\left(\left|M_{g, L} f\right|^{p} u\right)\left(T^{j} x\right) d \mu .
$$

Now, if $[-k-L, k+L]=\{j \in \mathbf{Z}:-k-L \leqslant j \leqslant k+L\}$, then the right-hand side in (3.11) is smaller than or equal to

$$
\int_{X}(2 k+1)^{-1} \sum_{j=-\infty}^{\infty}\left|m_{g^{x}}\left(f^{x} \chi_{[-k-L, k+L]}\right)(j)\right|^{p} u\left(T^{j} x\right) d \mu,
$$

where $f^{x}(i)=f\left(T^{i} x\right)$. Then, by Lemma (3.9), the integrand is dominated by

$$
C \sum_{j=-k-L}^{k+L}\left|f\left(T^{j} x\right)\right|^{p} w\left(T^{j} x\right) \text { a.e. }
$$

If we put these inequalities in (3.11) and keep in mind that $T$ preserves the measure $\mu$ we get

$$
\int_{X}\left|M_{g, L} f\right|^{p} u d \mu \leqslant C(2 k+1)^{-1}(2 k+2 L+1) \int_{X}|f|^{p} w d \mu .
$$

Then, if we let $k$ and then $L$ go to $\infty$ we obtain proposition (a) in Theorem (3.5).

4. Relation between $A_{p}^{\prime}(g, T, \mu)$ and $S_{p}^{\prime}(g, T, \mu)$. By a straightforward observation or by Theorems (2.7) and (3.5) it follows that if the pair $(u, w)$ satisfies $S_{p}^{\prime}(g, T, \mu)$, then $(u, w)$ satisfies $A_{p}^{\prime}(g, T, \mu)$. The other implication is not true as Example (3.1) 
and Theorems (2.7) and (3.5) show. However, in the same way as in the HardyLittlewood maximal operator, both conditions are equivalent if $u=w$.

(4.1) TheOREM. Let $(X, \mathscr{F}, \mu), T, g$, and $w$ be as in $\$ \S 2$ and 3. If $1<p<\infty$, then the following conditions are equivalent:

(a) There exists $C>0$ such that for all $\lambda>0$ and for every measurable function $f$

$$
\int_{\left\{x \in X: M_{g} f(x)>\lambda\right\}} w d \mu \leqslant C \lambda^{-p} \int_{X}|f|^{p} w d \mu .
$$

(b) There exists $C>0$ such that for every measurable function $f$

$$
\int_{X}\left|M_{g} f\right|^{p} w d \mu \leqslant C \int_{X}|f|^{p} w d \mu
$$

(c) $w$ satisfies $A_{p}^{\prime}(g, T, \mu)$.

(d) $w$ satisfies $S_{p}^{\prime}(g, T, \mu)$ (i.e. $(w, w)$ belongs to the $S_{p}^{\prime}(g, T, \mu)$ class $)$.

Proof. Once Theorems (2.7) and (3.5) have been established we have only to prove that $(\mathrm{c}) \Rightarrow(\mathrm{d})$. In order to prove this we shall follow the idea of [6]. Let $k$ be a nonnegative integer. Let us fix $i, 0 \leqslant i \leqslant k$, and let $r$ and $s$ be integers such that $0 \leqslant r \leqslant i$ and $0 \leqslant s \leqslant k-i$. Then, since $w$ satisfies $A_{p}^{\prime}(g, T, \mu)$, we have

$$
\begin{aligned}
T_{r, s}^{g}\left(w^{-1} g\right)^{q / p}\left(T^{i} x\right) \leqslant C\left[\sum_{j=-r}^{s} \chi_{[0, k]}(j+i)\left(w^{-1} g w\right)\left(T^{j+i} x\right)\right. \\
\left.\cdot\left[\sum_{j=-r}^{s} w\left(T^{j+i} x\right)\right]^{-1}\right]^{q / p}
\end{aligned}
$$

for almost all $x$ in $F=X-\bigcup_{j=-\infty}^{\infty} T^{j}(\{x \in X: w(x)=0\})$, where $[0, k]=\{j \in \mathbf{Z}$ : $0 \leqslant j \leqslant k\}$. Therefore

$$
M_{i, k-i}^{g}\left(w^{-1} g\right)^{q / p}\left(T^{i} x\right) \leqslant C\left[m_{w^{x}}\left(\left(w^{x}\right)^{-1} g^{x} \chi_{[0, k]}\right)(i)\right]^{q / p}
$$

for almost all $x$ in $F$ and where $w^{x}(j)=w\left(T^{j} x\right)$ and $g^{x}(j)=g\left(T^{j} x\right)$. Then, raising to $p$, multiplying by $w\left(T^{i} x\right)$ and summing in $i$, we get

$$
\begin{aligned}
& \sum_{i=0}^{k}\left|M_{i, k-i}^{g}\left(w^{-1} g\right)^{q / p}\left(T^{i} x\right)\right|^{p} w\left(T^{i} x\right) \\
& \leqslant C \sum_{i=0}^{k}\left|m_{w^{x}}\left(\left(w^{x}\right)^{-1} g^{x} \chi_{[0, k]}\right)(i)\right|^{q} w\left(T^{i} x\right)
\end{aligned}
$$

for almost all $x$ in $F$. Now, by (2.24) applied to the operators $m_{w^{x}}$, there exists a constant $C>0$ such that for almost all $x$ in $F$

$$
\sum_{i=0}^{k}\left|m_{w^{x}}\left(\left(w^{x}\right)^{-1} g^{x} \chi_{[0, k]}\right)(i)\right|^{q} w\left(T^{i} x\right) \leqslant C \sum_{i=0}^{k} g^{q}\left(T^{i} x\right) w^{-q / p}\left(T^{i} x\right)
$$


Then (4.4) and (4.5) give us

$$
\sum_{i=0}^{k}\left|M_{i, k-i}^{g}\left(w^{-1} g\right)^{q / p}\left(T^{i} x\right)\right|^{p} w\left(T^{i} x\right) \leqslant C \sum_{i=0}^{k}\left(g^{q} w^{-q / p}\right)\left(T^{i} x\right)
$$

for almost all $x$ in $F$. This proves (c) $\Rightarrow$ (d).

We have just shown that if we consider a nonnegative function $w$, then the operator $M_{g}$ is of strong type $(p, p)$ with respect to the measure $w d \mu$ if and only if $M_{g}$ is of weak type $(p, p)$ with respect to the same measure. Theorem (2.26) together with the last result allow us to get something else.

(4.6) Theorem. Let $(X, \mathscr{F}, \mu), T, g$, and $w$ be as in $\S \S 2$ and 3. If $1<p<\infty$ and $g$ satisfies (2.25), then the following conditions are equivalent:

(a) There exists $C>0$ such that for all $\lambda>0$ and for every measurable function $f$

$$
\sup _{k \geqslant 0} \int_{\left\{x \in X:\left|T_{k, k}^{g} f(x)\right|>\lambda\right\}} w d \mu \leqslant C \lambda^{-p} \int_{X}|f|^{p} w d \mu .
$$

(b) There exists $C>0$ such that for every measurable function $f$

$$
\int_{X}\left|M_{g} f\right|^{p} w d \mu \leqslant C \int_{X}|f|^{p} w d \mu .
$$

(c) $w$ satisfies $A_{p}^{\prime}(g, T, \mu)$.

(4.7) Note. Example (2.35) shows that Theorem (4.6) is not true if $g$ does not satisfy (2.25).

5. Finiteness of the maximal operator and convergence of the averages. It is well known that the almost everywhere convergence is closely related with the finiteness of the associated maximal operator. Actually, both problems are equivalent in some cases. In this section we will show that this equivalence holds in our setting.

(5.1) TheOREM. Let $(X, \mathscr{F}, \mu)$ be a $\sigma$-finite measure space and $T$ an invertible measure preserving transformation from $X$ onto itself. Finally let $g$ be a positive function in $L^{1}(d \mu)$ and let $w$ be a positive function. If $1<p<\infty$, then the following are equivalent:

(a) There exists a positive and measurable function $u: X \rightarrow \mathbf{R}$ such that the pair $(u, w)$ satisfies $S_{p}^{\prime}(g, T, \mu)$.

(b) $M_{g}\left(w^{-1} g\right)^{q / p}(x)<\infty$ a.e.

(c) There exists a positive and measurable function $u: X \rightarrow \mathbf{R}$ such that the pair (u,w) satisfies $A_{p}^{\prime}(g, T, \mu)$.

(d) $M_{g} f(x)<\infty$ a.e. for every $f$ in $L^{p}(w d \mu)$.

(e) The sequences $\left\{T_{0, k}^{g} f\right\}$ and $\left\{T_{k, 0}^{g} f\right\}$ are almost everywhere convergent for every $f$ in $L^{p}(w d \mu)$.

If $p=1$ statements $(\mathrm{c})-(\mathrm{e})$ are equivalent to

(f) $\sup _{-\infty<i<\infty}\left(w^{-1} g\right)\left(T^{i} x\right)<\infty$ a.e.

Proof of Theorem (5.1). Let $1<p<\infty$. It is clear that (a) $\Rightarrow$ (c) and (e) $\Rightarrow$ (d). Therefore, in order to prove the theorem it will suffice to show (c) $\Rightarrow(b),(b) \Rightarrow(d)$, (d) $\Rightarrow(\mathrm{c}),(\mathrm{b}) \Rightarrow(\mathrm{a})$, and (d) $\Rightarrow(\mathrm{e})$. In some of the implications we shall follow the idea of $[\mathbf{1 0}]$. 
(c) $\Rightarrow$ (b). By (c) there exists a constant $C>0$ such that for every pair $r, k$ of nonnegative integers and for almost all $x$ in $X$

$$
\left[T_{r, k}^{g}\left(w^{-1} g\right)^{q / p}(x)\right]^{p / q} \leqslant C\left[T_{r, k}^{g}\left(g^{-1} u\right)(x)\right]^{-1}=C T_{r, k}^{u}\left(u^{-1} g\right)(x)
$$

and so

$$
M_{g}\left(w^{-1} g\right)^{q / p}(x) \leqslant C\left[M_{u}\left(u^{-1} g\right)(x)\right]^{q / p} .
$$

Now, by Corollary (2.24), $M_{u}$ applies $L^{1}(u d \mu)$ into weak- $L^{1}(u d \mu)$ and since $u^{-1} g$ is in $L^{1}(u d \mu)$ we get that $M_{u}\left(u^{-1} g\right)(x)$ is finite for almost all $x$ in $X$. Putting this in (5.2) we have (b).

(b) $\Rightarrow(\mathrm{d})$. Let $f$ be a function in $L^{p}(w d \mu)$. By Hölder's inequality it is clear that

$$
M_{g} f(x) \leqslant\left[M_{g}\left(|f|^{p} w g^{-1}\right)(x)\right]^{1 / p}\left[M_{g}\left(w^{-1} g\right)^{q / p}(x)\right]^{1 / q} \text {. }
$$

By (b), the second factor of the right-hand side in the inequality is finite almost everywhere. On the other hand, the first factor is finite almost everywhere because $M_{g}$ applies $L^{1}(g d \mu)$ into weak- $L^{1}(g d \mu)$ (Corollary (2.24)). Therefore, $M_{g} f(x)<\infty$ a.e.

(d) $\Rightarrow$ (c). Since $M_{g}$ is the maximal operator associated to a family of positive operators, Nikishin's theorem [5] tells us that there exists a positive and measurable function $u$ from $X$ into $\mathbf{R}$ such that $M_{g}$ applies $L^{p}(w d \mu)$ into weak- $L^{p}(u w d \mu)$. Then, by Theorem (2.7), the pair ( $u w, w)$ satisfies $A_{p}^{\prime}(g, T, \mu)$.

(b) $\Rightarrow$ (a). It is clear that (a) is satisfied with

$$
u=\left(M_{g}\left(w^{-1} g\right)^{q / p}\right)^{-p} g^{q} w^{-q / p} .
$$

(d) $\Rightarrow($ e). Consider the conservative and the dissipative part, $\mathscr{C}$ and $\mathscr{D}$, associated to the transformation $T$. Remember (see [9] for example) that $X=\mathscr{C} \cup \mathscr{D}$ and since $g$ is in $L^{1}(d \mu)$ we have

$$
\begin{gathered}
\sum_{i=0}^{\infty} g\left(T^{i} x\right)=\infty \quad \text { for almost all } x \text { in } \mathscr{C}, \\
0<\sum_{i=0}^{\infty} g\left(T^{i} x\right)<\infty \quad \text { for almost all } x \text { in } \mathscr{D} .
\end{gathered}
$$

Let us fix $f$ in $L^{p}(w d \mu)$. By Theorem 4 in [13], there exists $\lim _{k \rightarrow \infty} T_{0, k}^{g} f(x)$ a.e. in $\mathscr{C}$ since $M_{g} f(x)<\infty$. In the dissipative part we know that $\sum_{i=0}^{\infty} g\left(T^{i} x\right)<\infty$ a.e. and since $M_{g} f(x)<\infty$ a.e. in $X$ we have $\sum_{i=0}^{\infty}\left|f\left(T^{i} x\right)\right| g\left(T^{i} x\right)<\infty$ for almost all $x$ in $\mathscr{D}$. Therefore

$$
\lim _{k \rightarrow \infty} T_{0, k}^{g} f(x)=\sum_{i=0}^{\infty} f\left(T^{i} x\right) g\left(T^{i} x\right)\left[\sum_{i=0}^{\infty} g\left(T^{i} x\right)\right]^{-1}
$$

for almost all $x$ in $\mathscr{D}$. Therefore, there exists $\lim _{k \rightarrow \infty} T_{0, k}^{g} f(x)$ a.e. $x$ in $X$. In the same way the existence of $\lim _{k \rightarrow \infty} T_{k, 0}^{g} f(x)$ a.e. is proved and hence we have proved (d) $\Rightarrow(\mathrm{e})$. 
Now let $p=1$. The equivalence $(\mathrm{d}) \Leftrightarrow(\mathrm{e})$ and the implication $(\mathrm{d}) \Rightarrow(\mathrm{c})$ are proved as in the case $p>1$. Hence we shall only prove (c) $\Rightarrow(f)$ and (f) $\Rightarrow(d)$.

(c) $\Rightarrow$ (f). Let $i$ be an integer. Since the pair $(u, w)$ satisfies $A_{1}^{\prime}(g, T, \mu)$ we have

$$
\left(w^{-1} g\right)\left(T^{i} x\right) \leqslant C\left[T_{|i|,|i|}^{g}\left(g^{-1} u\right)(x)\right]^{-1}=C T_{|i|,|i|}^{u}\left(u^{-1} g\right)(x) \text {. }
$$

Then, $\sup _{-\infty<i<\infty}\left(w^{-1} g\right)\left(T^{i} x\right) \leqslant C M_{u}\left(u^{-1} g\right)(x)$. Since $u^{-1} g$ is in $L^{1}(u d \mu)$ and $M_{g}$ applies $L^{1}(u d \mu)$ into weak- $L^{1}(u d \mu)$ we have $M_{u}\left(u^{-1} g\right)(x)<\infty$ a.e. Therefore $\sup _{-\infty<i<\infty}\left(w^{-1} g\right)\left(T^{i} x\right)<\infty$ a.e.

(f) $\Rightarrow(\mathrm{d})$. Let $f$ be in $L^{1}(w d \mu)$. It is clear that

$$
M_{g} f(x) \leqslant M_{g}\left(g^{-1} f w\right)(x) \sup _{-\infty<i<\infty}\left(w^{-1} g\right)\left(T^{i} x\right) .
$$

The second factor of the right-hand side in the inequality is finite a.e. in $X$ by (f). The first factor is also finite a.e. since $M_{g}$ applies $L^{1}(g d \mu)$ into weak $L^{1}(g d \mu)$. Therefore $M_{g} f(x)<\infty$ a.e.

(5.3) Note. Observe that propositions (a) and (c) are equivalent to $\left(a^{\prime}\right)$ and $\left(\mathrm{c}^{\prime}\right)$ by Theorems (2.7) and (3.5):

(a') There exist a constant $C>0$ and a positive measurable function $u: X \rightarrow \mathbf{R}$ such that

$$
\int_{X}\left|M_{g} f\right|^{p} u d \mu \leqslant C \int_{X}|f|^{p} w d \mu
$$

for every measurable function $f$.

(c') There exist a constant $C>0$ and a positive measurable function $u: X \rightarrow \mathbf{R}$ such that

$$
\int_{\left\{x \in X: M_{g} f(x)>\lambda\right\}} u d \mu \leqslant C \lambda^{-p} \int_{X}|f|^{p} w d \mu
$$

for all $\lambda>0$ and for every measurable function $f$.

Now, we shall use the last theorem to study mean convergence of the averages.

(5.4) ThEOREM. Let $(X, \mathscr{F}, \mu), T, g$, and $w$ be as in Theorem (5.1). If $g$ satisfies (2.25) and $1<p<\infty$, then the following are equivalent:

(a) $w$ satisfies $A_{p}^{\prime}(g, T, \mu)$.

(b) The sequences $\left\{T_{0, k}^{g} f\right\}$ and $\left\{T_{k, 0}^{g} f\right\}$ converge in $L^{p}(w d \mu)$ for every $f$ in $L^{p}(w d \mu)$.

If $g$ satisfies (2.25) and $w \in L^{1}(d \mu)$, then (a) and (b) are equivalent also for $p=1$.

Proof. It follows from (b) that the operators $T_{k, k}^{g}$ are uniformly bounded in $L^{p}(w d \mu)$. Then, by Theorem $(2.26), w$ satisfies $A_{p}^{\prime}(g, T, \mu)$.

Let $1<p<\infty$ and let us assume (a). By Theorem (4.1), $M_{g}$ is of strong type $(p, p)$. On the other hand, Theorem (5.1) tells us that $T_{0, k}^{g} f$ and $T_{k, 0}^{g} f$ are a.e. convergent for every $f$ in $L^{p}(w d \mu)$. Then (b) follows from Lebesgue's dominated convergence theorem.

Let us assume (a) with $p=1$ and $w \in L^{1}(d \mu)$. Since $A_{1}^{\prime}(g, T, \mu)$ implies $A_{2}^{\prime}(g, T, \mu)$ we get, by what we have already shown, that $T_{0, k}^{g} f$ and $T_{k, 0}^{g} f$ converge in $L^{2}(w d \mu)$ for every $f$ in $L^{2}(w d \mu)$. Since $w \in L^{1}(d \mu)$, the sequences of averages 
converge in $L^{1}(w d \mu)$ for every $f$ in $L^{2}(w d \mu)$. Now, (b) follows from this because $L^{2}(w d \mu)$ is a dense subset of $L^{1}(w d \mu)$ and the averages are uniformly bounded in $L^{1}(w d \mu)$ (Theorem (2.26)).

Theorems (5.1) and (5.4) can be used to solve the problem stated in the introduction about the convergence of Hopf's averages. The results follow from (5.1), (5.4) and (4.6) if we remember that $D_{r, k}^{g} f=T_{r, k}^{g}\left(g^{-1} f\right), \mathscr{M}_{g} f=M_{g}\left(g^{-1} f\right)$ and keep in mind that $f \in L^{p}(w d \mu)$ if and only if $g^{-1} f \in L^{p}\left(g^{p} w d \mu\right)$.

(5.5) Theorem. Let $(X, \mathscr{F}, \mu), T, g$, and $w$ be as in Theorem (5.1). If $1<p<\infty$, the following are equivalent:

(a) There exists a positive and measurable function $u: X \rightarrow \mathbf{R}$ such that $\left(u, g^{p} w\right)$ satisfies $S_{p}^{\prime}(g, T, \mu)$.

(b) There exist a constant $C>0$ and a positive and measurable function $u: X \rightarrow \mathbf{R}$ such that

$$
\int_{X}\left|\mathscr{M}_{g} f\right|^{p} u d \mu \leqslant C \int_{X}|f|^{p} w d \mu
$$

for every measurable function $f$.

(c) There exists a positive and measurable function $u: X \rightarrow \mathbf{R}$ such that $\left(u, g^{p} w\right)$ satisfies $A_{p}^{\prime}(g, T, \mu)$.

(d) There exist a constant $C>0$ and a positive measurable function $u: X \rightarrow \mathbf{R}$ such that

$$
\int_{\left\{x \in X: \mathscr{M}_{g} f(x)>\lambda\right\}} u d \mu \leqslant C \lambda^{-p} \int_{X}|f|^{p} w d \mu
$$

for each $\lambda>0$ and for every measurable function $f$.

(e) $\mathscr{M}_{g}\left(w^{-q / p}\right)(x)<\infty$ a.e.

(f) $\mathscr{M}_{g} f(x)<\infty$ a.e. for every $f$ in $L^{p}(w d \mu)$.

(g) The sequences $\left\{D_{0, k}^{g} f\right\}$ and $\left\{D_{k, 0}^{g} f\right\}$ are a.e. convergent for every $f$ in $L^{p}(w d \mu)$.

If $p=1,(\mathrm{c}),(\mathrm{d}),(\mathrm{f})$, and $(\mathrm{g})$ are equivalent to

(h) $\sup _{-\infty<i<\infty} w^{-1}\left(T^{i} x\right)<\infty$ a.e.

(5.6) ThEOREM. Let $(X, \mathscr{F}, \mu), T, g$, and $w$ be as in Theorem (5.1). If $g$ satisfies (2.25) and $1<p<\infty$, the following are equivalent:

(a) $g^{p} w$ satisfies $A_{p}^{\prime}(g, T, \mu)$.

(b) There exists a constant $C>0$ such that

$$
\int_{X}\left|\mathscr{M}_{g} f\right|^{p} g^{p} w d \mu \leqslant C \int_{X}|f|^{p} w d \mu
$$

for every measurable function $f$.

(c) There exists a constant $C>0$ such that

$$
\sup _{k \geqslant 0} \int_{\left\{x \in X: D_{k, k}^{g} f(x)>\lambda\right\}} g^{p} w d \mu \leqslant C \lambda^{-p} \int_{X}|f|^{p} w d \mu
$$

for each $\lambda>0$ and for every fin $L^{p}(w d \mu)$. 
(d) The sequences $\left\{D_{0, k}^{g} f\right\}$ and $\left\{D_{k, 0}^{g} f\right\}$ converge in $L^{p}\left(g^{p} w d \mu\right)$ for every measurable function in $L^{p}(w d \mu)$.

If $g$ satisfies (2.25), $p=1$ and $w \in L^{1}(d \mu)$, then $(\mathrm{a}),(\mathrm{c})$, and $(\mathrm{d})$ are equivalent to (e) and (f):

(e) There exist a constant $C>0$ such that

$$
\int_{\left\{x \in X: \mathscr{M}_{g} f(x)>\lambda\right\}} g w d \mu \leqslant C \lambda^{-1} \int_{X}|f| w d \mu
$$

for each $\lambda>0$ and for every measurable function $f$.

(f) There exists a constant $C>0$ such that

$$
\sup _{k \geqslant 0} \int_{X}\left|D_{k, k}^{g} f\right| g w d \mu \leqslant C \int_{X}|f| w d \mu
$$

for every measurable function $f$.

(5.7) Note. As in (5.5) and (5.6), theorems in $\$ \S 2-4$ can be written for the maximal operator $\mathscr{M}_{g}$ and the averages $D_{r, k}^{g}$. It is also possible to establish the theorems of this paper for the general averages

$$
\sum_{i=-r}^{k}(f h)\left(T^{i} x\right)\left[\sum_{i=-r}^{k} g\left(T^{i} x\right)\right]^{-1}
$$

where $h$ and $g$ are positive and measurable functions. For this, it suffices to observe that the averages in (5.8) are $T_{r, k}^{g}\left(g^{-1} f h\right)$ and keep in mind that $f \in L^{p}(w d \mu)$ if and only if $g^{-1} f h \in L^{p}\left(g^{p} h^{-p} w d \mu\right)$.

Finally, we shall establish the results when $g(x)=1$ and $\mu(X)<\infty$.

(5.9) Corollary. Let $(X, \mathscr{F}, \mu)$ be a finite measure space and $T$ an invertible measure preserving transformation from $X$ into itself. Let $w: X \rightarrow \mathbf{R}$ be a positive and measurable function. If $1<p<\infty$, then the following are equivalent:

(a) The sequences $T_{0, k} f(x)=(k+1)^{-1} \sum_{i=0}^{k} f\left(T^{i} x\right)$ and $T_{k, 0} f(x)=$ $(k+1)^{-1} \sum_{i=0}^{k} f\left(T^{-i} x\right)$ converge a.e. for every $f \in L^{p}(w d \mu)$.

(b) The sequence $(2 k+1)^{-1} \sum_{i=-k}^{k} f\left(T^{i} x\right)$ is a.e. convergent for every $f \in L^{p}(w d \mu)$.

(c) $\sup _{r, k \geqslant 0}(r+k+1)^{-1} \sum_{i=-r}^{k} w^{-q / p}\left(T^{i} x\right)<\infty$ a.e.

If $p=1$, (a) and (b) are equivalent to

(d) $\sup _{-\infty<i<\infty} w^{-1}\left(T^{i} x\right)<\infty$ a.e.

Proof of Corollary (5.9). Let $1<p<\infty$. By Theorem (5.1), (a) and (c) are equivalent. It is easy to see that (a) implies (b). On the other hand, it follows from (b) that

$$
\sup _{k \geqslant 0}(2 k+1)^{-1} \sum_{i=-k}^{k}|f|\left(T^{i} x\right)<\infty \quad \text { a.e. for every } f \in L^{p}(w d \mu) .
$$

Then

$$
\sup _{r, k \geqslant 0}(r+k+1)^{-1} \sum_{i=-r}^{k}|f|\left(T^{i} x\right)<\infty \quad \text { a.e. }
$$

for each $f \in L^{p}(w d \mu)$. Now, we get (c) by Theorem (5.1).

If $p=1$ the proof is similar. 
(5.10) Corollary. Let $(X, \mathscr{F}, \mu)$ be a finite measure space and $T$ an invertible measure preserving transformation from $X$ into itself. Let $w: X \rightarrow \mathbf{R}$ be a positive and measurable function. If $1<p<\infty$, then the following are equivalent:

(a) The sequences $\left\{T_{0, k} f\right\}$ and $\left\{T_{k, 0} f\right\}$ converge in $L^{p}(w d \mu)$ for every $f \in$ $L^{p}(w d \mu)$.

(b) The sequence $T_{k, k} f(x)=(2 k+1)^{-1} \sum_{i=-k}^{k} f\left(T^{i} x\right)$ is convergent in $L^{p}(w d \mu)$ for every $f \in L^{p}(w d \mu)$.

(c) $w$ satisfies $A_{p}^{\prime}(T, \mu)$.

If $p=1$ and $w \in L^{1}(d \mu)$, then (a)-(c) are also equivalent.

Proof of Corollary (5.10). Let $1<p<\infty$. Statements (a) and (c) are equivalent by Theorem (5.4). It is easy to see that (a) implies (b). Let us assume (b). Then operators $T_{k, k}$ are uniformly bounded in $L^{p}(w d \mu)$. Now (c) follows from Theorem (2.26).

If $p=1$ the proof is similar.

(5.11) Note. Corollary (5.10) is true even if $\mu(X)=\infty$ (it follows from VIII.5.4 and VIII.5.5 in [4]; the case $p>1$ can be found in [7]). However, if $p=1$, $\mu(X)=\infty$ and $w \notin L^{1}(d \mu)$ is false. In order to observe this, let $X=\mathbf{Z}$, F the power set of $\mathbf{Z}, \mu$ the counting measure, $T(i)=i+1$ and $w(i)=1$. It is clear that $w$ satisfies $A_{1}^{\prime}(T, \mu)$ and $\lim T_{0, k} f(x)=\lim T_{k, 0} f(x)=\lim T_{k, k} f(x)=0$ a.e. for every $f \in L^{1}(w d \mu)$. However, (a) and (b) do not hold in this example since

$$
\lim \int_{X}\left(T_{0, k} f\right) w d \mu=\lim \int_{X}\left(T_{k, 0} f\right) w d \mu=\lim \int_{X}\left(T_{k, k} f\right) w d \mu=\int_{X} f w d \mu .
$$

6. Higher dimensional results. Let $(X, \mathscr{F}, \mu)$ be a $\sigma$-finite measure space and let $g$ be a positive measurable function. We shall consider a group $\left\{T^{i}: i \in \mathbf{Z}^{n}\right\}$ of invertible measure preserving transformations from $X$ into itself. For each cube $I$ in $\mathbf{Z}^{n}$ such that $0 \in I$ we define the averages $T_{I}^{g}$ by

$$
T_{I}^{g} f(x)=\left[\sum_{i \in I}(f g)\left(T^{i} x\right)\right]\left[\sum_{i \in I} g\left(T^{i} x\right)\right]^{-1}
$$

and the maximal operator $M_{g}$ associated to these averages (for $n=1$ we are in the setting of the previous sections). Then it is natural to ask the same questions as in the case $n=1$. For example, when is it true that $M_{g}$ is of weak type with respect to the measures $w d \mu$ and $u d \mu$ ? It is possible to prove almost all the results if $g$ satisfies a "doubling condition" over cubes in $\mathbf{Z}^{n}$, i.e., if there exists a constant $C>0$ such that for every nonnegative integer $k$

$$
\sum_{i \in J_{k}} g\left(T^{i} x\right) \leqslant C \sum_{i \in I_{k}} g\left(T^{i} x\right) \text { a.e. }
$$

with $I_{k}=[0, k] \times[0, k] \times \cdots \times[0, k](n$ times $), J_{k}=[-k, 2 k] \times \cdots \times[-k, 2 k](n$ times) and $[r, h]=\{j \in \mathbf{Z}: r \leqslant j \leqslant h\}$. So, Theorem (2.26) holds where $A_{p}^{\prime}$ is the following condition:

$$
\begin{gathered}
M_{g}\left(g^{-1} u\right)(x) \leqslant C\left(g^{-1} w\right)(x) \quad \text { a.e. for } p=1, \\
\sup _{k \geqslant 0} T_{I_{k}}^{g}\left(g^{-1} u\right)(x)\left[T_{I_{k}}^{g}\left(w^{-1} g\right)^{q / p}(x)\right]^{p / q} \leqslant C \quad \text { a.e. for } 1<p<\infty .
\end{gathered}
$$


If the pair $(u, w)$ satisfies the $S_{p}^{\prime}$ condition over cubes in $\mathbf{Z}^{n}$, then $M_{g}$ is of strong type $(p, p)(1<p<\infty)$. We do not know if the converse is true for $n>1$. However the converse holds if we are in any of the following general cases:

(a) $T^{i}$ is ergodic for every $i \neq 0$ such that the greatest common divisor of $\left\{i_{h} \neq 0\right\}$ is equal to 1 , where $i=\left(i_{1}, i_{2}, \ldots, i_{n}\right)$.

(b) $(X, \mathscr{F}, \mu)$ is the product space of $\left(X_{j}, \mathscr{F}_{j}, \mu_{j}\right), j=1, \ldots, n$, and $T^{i} x=$ $\left(T_{1}^{i_{1}} x_{1}, \ldots, T_{n}^{\left.i_{n} x_{n}\right)}\right.$ where $i=\left(i_{1}, \ldots, i_{n}\right), x=\left(x_{1}, \ldots, x_{n}\right)$ and $T_{j}: X_{j} \rightarrow X_{j}$ is an invertible transformation that preserves the measure $\mu_{j}$.

But in all the cases and always assuming that $g$ satisfies a "doubling condition" we get that strong and weak type $(p, p)(1<p<\infty)$ are equivalent if $u=w$. The keys of these results are transference arguments and an $n$-dimensional lemma that plays the role of (2.10) which is obtained from (2.9).

FinAL REMARK. Using the ideas of [3] and assuming that $g$ satisfies a "doubling condition" it can be proved that a positive function $w$ is in $A_{p}^{\prime}(g, T, \mu)$ if and only if there exist $w_{0}$ and $w_{1}$ satisfying $A_{1}^{\prime}(g, T, \mu)$ and such that $w=g^{p-1} w_{0} w_{1}^{1-p}$ (the case $n=1$ and $g(x)=1$ can be found in [7]). For $n=1$, this factorization holds even if $g$ does not satisfy a "doubling condition".

\section{REFERENCES}

1. E. Atencia and F. J. Martin-Reyes, Weak type inequalities for the maximal ergodic function and the maximal ergodic Hilbert transform in weighted spaces, Studia Math. 78 (1984), 231-244.

2. E. Atencia and A. de la Torre, A dominated ergodic estimate for $L_{p}$ spaces with weights, Studia Math. 74 (1982), 35-47.

3. R. Coifman, Peter W. Jones and José L. Rubio de Francia, On a constructive decomposition of BMO functions and factorizations of $A_{p}$ weights, Proc. Amer. Math. Soc. 87 (1983), 675-676.

4. N. Dunford and J. T. Schwartz, Linear operators, Part I, Interscience, New York, 1957.

5. J. E. Gilbert, Nikishin-Stein theory and factorization with applications, Proc. Sympos. Pure Math., vol. 35. Amer. Math. Soc., Providence, R. I., 1979, pp. 233-267.

6. R. Hunt, D. Kurtz and C. Neugebauer, $A$ note on the equivalence of $A_{p}$ and Sawyer's condition for equal weights, Conference on Harmonic Analysis in honor of Antoni Zygmund, Vols. I, II (Chicago, Ill., 1981), Wadsworth Math. Ser., Wadsworth, Belmont, Calif., 1983, pp. 156-158.

7. F. J. Martin-Reyes and A. de la Torre, $A$ dominated ergodic theorem for invertible $L_{p}$-isometries in weighted $L_{p}$-spaces (preprint).

8. B. Muckenhoupt, Weighted norm inequalities for the Hardy maximal function, Trans. Amer. Math. Soc. 165 (1972), 207-226.

9. D. Revuz, Markov chains, North-Holland Mathematical Library, 1975.

10. J. L. Rubio de Francia, Boundedness of maximal functions and singular integrals in weighted $L^{p}$ spaces, Proc. Amer. Math. Soc. 83 (1981), 673-679.

11. E. T. Sawyer, A characterization of a two-weight norm inequality for maximal operators, Studia Math. 75 (1982), 1-11.

12. __ Two weight norm inequalities for certain maximal and integral operators, Harmonic Analysis, Proceedings, Minneapolis, 1981, Springer-Verlag, Berlin and New York, 1982, pp. 102-127.

13. J. Woś, The filling scheme and the ergodic theorems of Kesten and Tanny, Colloq. Math. (to appear).

Departamento de Analisis Matematico, Facultad de Ciencias, Universidad de Málaga, Colonia de Santa Intes, Málaga, Spain 